\title{
Transverse beam dynamics in a plasma density ramp
}

\author{
R. Ariniello, ${ }^{1,}{ }^{*}$ C. E. Doss, ${ }^{1}$ K. Hunt-Stone, ${ }^{1}$ J. R. Cary,${ }^{1,2}$ and M. D. Litos ${ }^{1}$ \\ ${ }^{1}$ Center for Integrated Plasma Studies, Department of Physics, University of Colorado Boulder, \\ Boulder, Colorado 80309, USA \\ ${ }^{2}$ Tech-X Corporation, Boulder, Colorado 80301, USA
}

(Received 21 December 2018; published 30 April 2019)

\begin{abstract}
An electron beam experiences chromatic emittance growth in a plasma-based accelerator if it is not matched to the focusing force in the plasma wake. A ramped plasma density profile at the entrance and exit of the plasma source can control the focusing of the beam into and out of the plasma accelerator, limiting emittance growth. Here, we present a comprehensive, analytic theory to describe the transverse beam dynamics and emittance growth in a nearly arbitrary plasma ramp profile. For a given incoming beam, this theory can be used to determine the length of the ramp required to correctly focus the electron beam, the optimal location of the beam's vacuum focus, and the chromatic emittance growth in the ramp. In addition, the theory can be used to determine the effect that errors in the beam focusing and plasma profile have on the emittance of the beam. We illustrate two example ramps to demonstrate the theory: one that provides very fast focusing for beam matching, and one that is robust to errors in the plasma density profile.
\end{abstract}

DOI: 10.1103/PhysRevAccelBeams.22.041304

\section{INTRODUCTION}

Modern, high energy particle accelerators powered by radio frequency (rf) klystrons are becoming increasingly large and expensive. Plasma based accelerators are a compact alternative capable of producing accelerating gradients hundreds to thousands of times larger than conventional rf accelerators, reducing the accelerator footprint by a corresponding factor. In a plasma-based accelerator, a laser or particle beam drives an electron density wave in the plasma, called a wake, producing a strong, longitudinal electric field. A second electron beam placed within the wake - the witness beam-is accelerated by the electric field, gaining significant energy in a short distance. Recent experiments have shown that an electron beam driven plasma wakefield accelerator (PWFA) can produce accelerating gradients in excess of $10 \mathrm{GeV} / \mathrm{m}$ with high efficiency and low energy spread [1-3]. Laser driven wakefield accelerators (LWFA) have demonstrated accelerating gradients as high as $100 \mathrm{GeV} / \mathrm{m}$, producing beams with energy up to $4.2 \mathrm{GeV}$ [4,5]. To be suitable for applications, however, a plasma-based accelerator must preserve the beam quality, characterized by the beam's transverse emittance, during acceleration. After acceleration, the beam must be coupled into a conventional

\footnotetext{
*robert.ariniello@colorado.edu
}

Published by the American Physical Society under the terms of the Creative Commons Attribution 4.0 International license. Further distribution of this work must maintain attribution to the author(s) and the published article's title, journal citation, and DOI. magnetic beam line for delivery to the interaction point or another accelerating stage. So far, high-gradient PWFA and LWFA experiments have failed to preserve the emittance of the beam.

In a plasma accelerator operating in the highly nonlinear blowout regime, all of the plasma electrons are evacuated from the center of the wake. The ions remain in place due to their inertia, resulting in a bare ion column inside the wake with a density equal to the initial plasma density. The electric field of the ion column exerts a transverse focusing force on the beam electrons. Energy spread in the electron beam drives emittance growth when the beam's divergence is not matched to the focusing strength of the plasma. The beam size required for matching to the plasma in a plasmabased accelerator is much smaller than that typically encountered in a conventional accelerator and is beyond the capabilities of most magnetic electron beam optics. Because the beam is so highly focused in the plasma, it has a large divergence. When the beam exits the plasma, its divergence is no longer balanced by the focusing force of the plasma and the beam undergoes rapid expansion. This expansion can lead to catastrophic normalized emittance growth and beam loss due to overfilling of apertures in the downstream magnetic beam line [6-8].

Since the focusing force of the ion column depends on the plasma density, a longitudinally tapered density profile can perform the focusing necessary to match the witness beam into and out of the plasma accelerator [9-11]. Figure 1 shows a conceptual example of this scheme. A conventional magnetic optic focuses the beam into a ramped plasma density profile. The ion column in the ramp focuses the beam further such that it is matched to the plasma as it enters 


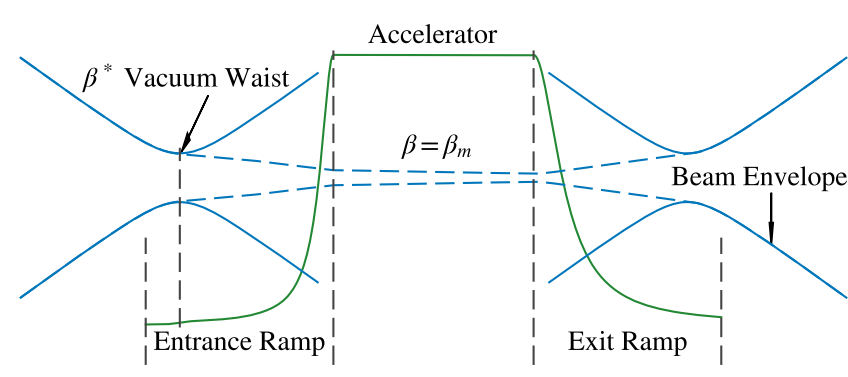

FIG. 1. A conceptual diagram showing plasma density ramps coupling a beam into and out of a plasma-based accelerator. The electron beam propagates from left to right, with a vacuum waist near the plasma entrance ramp location. The solid blue lines indicate the envelope of the beam as it would propagate through vacuum, and the dashed blue lines indicate the envelope of the beam as it would propagate through the plasma. The plasma density profile is shown in green. The plasma entrance ramp focuses the electron beam to the matched size as it enters the accelerator, and the plasma exit ramp reduces the divergence of the electron beam as it leaves the accelerator. The beam gains energy in the accelerator, leading to adiabatic damping and necessitating an exit ramp that is longer than the entrance ramp.

the accelerating region. A similar ramp placed at the exit of the accelerating region gradually increases the size of the beam, controlling its divergence as it exits the plasma. This allows the beam to couple into a magnetic beam transport system.

Previous literature on transverse beam dynamics in a longitudinal plasma ramp by Chen et al. [12] considered adiabatic focusing in a slowly changing plasma density in the context of exceeding the Oide limit. Floettmann [10] and $\mathrm{Xu}$ et al. [11] showed that several plasma density profiles are theoretically capable of matching a beam into a plasma, though they treat only a small set of ramp shapes where the transverse beam evolution is analytically solvable. In addition, all of the ramp profiles they consider have a nonphysical discontinuous first derivative at the location where the ramp joins the uniform density section of the plasma used for acceleration. Similar dynamics occur for a laser pulse passing through a plasma ramp at the entrance of a plasma channel [13]. However, the effect that the ramp shape has on the focusing of the beam and the emittance growth within the ramp have not yet been considered.

In this work, we present a theoretical description of the transverse beam evolution in an arbitrarily shaped plasma ramp. In doing so, we divide the plasma ramp into three different regimes: an adiabatic regime, where the plasma density changes slowly on the betatron oscillation scale; a nonadiabatic regime, where the plasma density changes rapidly; and a perturbation regime, where the plasma density is low and only perturbs the beam evolution away from vacuum propagation. We present a complete analytic theory describing the beam evolution in an adiabatic or perturbative plasma ramp, and a limited analytic treatment of a nonadiabatic plasma ramp. Using the solutions presented here, the ramp length, the location of the electron beam's vacuum waist, and the evolution of the beam's projected emittance can be calculated directly. The theory can be used to design plasma density ramps for matching a given beam into or out of a plasma accelerator. It can also be used to calculate the effects of variations in beam or plasma parameters on the beam's emittance growth. We demonstrate how the theory can be used to design an entrance ramp for a plasma based accelerator and predict the emittance evolution for a beam with incoming chromatic effects. Further, we show that rapid focusing occurs for a highly mismatched beam entering a uniform density plasma, producing a very short ramp that can match the beam into the accelerating section.

\section{FOCUSING IN A PLASMA BASED ACCELERATOR}

A laser or electron driver passing through an underdense plasma will excite a nonlinear wake that expels the plasma electrons, leaving a bare ion column. The witness beam experiences a linear focusing force inside the column. The transverse equation of motion for an electron in the beam is

$$
\frac{d^{2} x}{d s^{2}}+K(s) x=0
$$

where $s$ is the longitudinal coordinate in the direction of beam propagation, and $x$ is the transverse position of the particle. The focusing strength of the plasma is given by

$$
K(s)=\frac{\omega_{p}^{2}(s)}{2 \gamma_{b} c^{2}},
$$

where $\omega_{p}$ is the plasma frequency defined as $\omega_{p}(s)^{2}=$ $n(s) e^{2} / m_{e} \epsilon_{0}, n(s)$ is the plasma density, $e$ is the elementary charge, $m_{e}$ is the electron mass, $\epsilon_{0}$ is the permittivity of free space, $\gamma_{b}$ is the relativistic factor of the particle, and $c$ is the speed of light. The length scale of transverse motion in the plasma is set by the betatron wave number, defined as $k_{\beta}^{2}(s)=K(s)$. in this work, we consider ramps leading into and out of a uniform density plasma. It is convenient to use $1 / k_{\beta}$ at the beginning (end) of the uniform plasma, denoted $1 / k_{\beta 0}$, as the unit of length for an entrance (exit) ramp. From here on, all quantities with units of length are written in units of $1 / k_{\beta 0}$. Further, we define $\eta=n / n_{0}$, where $n_{0}$ is the density of the uniform plasma. In these units $k_{\beta}(s)=\sqrt{\eta(s)}$. The focusing strength is written as $K(s)=\eta(s)$.

One measure of the beam quality as a whole is the normalized emittance defined as $\epsilon_{n}=\left(1 / m_{e} c\right) \times$ $\sqrt{\left\langle x^{2}\right\rangle\left\langle p_{x}^{2}\right\rangle-\left\langle x p_{x}\right\rangle^{2}}$, where $p_{x}$ is the transverse momentum of the particle [11]. The normalized emittance is usually written as $\epsilon_{n}=\left\langle\gamma_{b}\right\rangle \epsilon$, where $\left\langle\gamma_{b}\right\rangle$ is the average relativistic factor of the beam [6,7]. We assume there is 
negligible relative energy change in the ramp $\Delta \gamma_{b} / \gamma_{b} \ll 1$, where $\Delta \gamma_{b}$ is the change in beam energy within the ramp. Under this assumption the relative growth in normalized emittance is equivalent to the relative growth in geometric emittance $\epsilon=\sqrt{\left\langle x^{2}\right\rangle\left\langle x^{\prime 2}\right\rangle-\left\langle x x^{\prime}\right\rangle^{2}}$. Here $x^{\prime}=d x / d s=$ $p_{x} / p_{s}$. The transverse phase space of the beam is parametrized by the Courant-Snyder parameters, where $\beta=$ $\left\langle x^{2}\right\rangle / \epsilon$ measures the squared width of the beam, $\gamma=$ $\left\langle x^{\prime 2}\right\rangle / \epsilon$ gives the squared divergence, and $\alpha=-\left\langle x x^{\prime}\right\rangle / \epsilon$ is the correlation between the particle position and divergence. The evolution of $\beta$ is described by [14]

$$
\frac{1}{2} \beta^{\prime \prime}+k_{\beta}^{2}(s) \beta-\frac{1}{\beta}\left[1+\left(\frac{1}{2} \beta^{\prime}\right)^{2}\right]=0,
$$

which cannot in general be solved for arbitrary $k_{\beta}(s)$.

Emittance growth in a plasma accelerator arises from chromatic dephasing of the beam in the ion column. Chromatic effects are parametrized by the fractional momentum deviation $\delta$ defined as $\delta=\left(p-p_{0}\right) / p_{0}$, where $p_{0}$ is the reference particle's momentum. In this work, we assume $\delta \ll 1$. The energy dependence of the focusing strength in the highly relativistic approximation is given by

$$
K(s, \delta)=\frac{\eta(s)}{1+\delta}
$$

The energy dependence of the betatron wave number is $k_{\beta}(s, \delta)=\sqrt{\eta /(1+\delta)}$ and the betatron evolution is described by Eq. (3), where $k_{\beta}$ is an explicit function of $\delta$. The energy dependent focusing force leads to emittance growth for any beam with finite energy spread. Figure 2

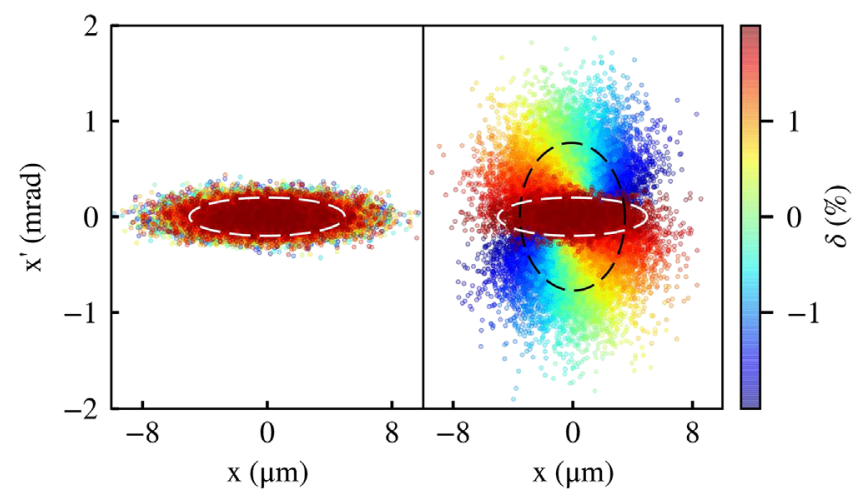

FIG. 2. Emittance growth due to chromatic dephasing, the initial phase space distribution is shown on the left and the distribution after the beam propagates 23.7 betatron oscillations is shown on the right. The initial rms phase space ellipse is shown in white and the final rms phase space ellipse is shown in black. The ellipses are magnified by a factor of 2 for clarity. The area of the ellipse is 4 times the rms emittance, the final emittance is a factor of 2.5 larger than the initial emittance due to the energy spread in the beam. shows the transverse phase space of an unmatched beam before and after propagating through a uniform density plasma. Different energy slices of the beam initially overlap in phase space. As the beam propagates, the slices rotate at different rates due to the energy dependence of the focusing force, spreading the beam out in transverse phase space. This chromatic dephasing leads to growth in the projected beam emittance. The area of the white ellipse in the figure is the initial emittance and the area of the black ellipse is the final emittance, about 2.5 times larger.

Chromatic emittance growth is minimized if the beam starts matched to the plasma. In a uniform density plasma, a monoenergetic beam is considered matched if its transverse envelope does not change size as it propagates through the plasma. Thus, for a matched beam, $\beta$ must be constant and therefore $\alpha=0$. This condition is satisfied when $\beta=1 / k_{\beta}$. In a nonuniform plasma, we define the matched beta function $\beta_{m}(s)=1 / k_{\beta}(s)$. Further, we define $\alpha_{m}=-\beta_{m}^{\prime} / 2$, analogous to the definition of the CourantSnyder parameter $\alpha$. Minimal emittance growth occurs in the uniform accelerating region of a plasma accelerator when the beam is matched to the plasma according to its average energy $\left\langle\gamma_{b}\right\rangle$ [11]. A longitudinally tapered plasma density ramp is designed to focus a beam to the matched size as it enters the accelerating section.

\section{ADIABATIC EVOLUTION}

In an adiabatic ramp, the plasma density changes slowly compared to the length scale of the beam's betatron oscillations. Focusing of a beam using a slowly changing plasma density has been considered before [8,10,12,15-17]. However, the full solution for the beam dynamics, including the evolution of a mismatched beam, has not been explicitly derived. The adiabatic solution is an expansion in $\alpha_{m}$; accordingly, we define the adiabaticity parameter as $\mathcal{A}=\left|\alpha_{m}\right|$. The adiabatic limit is defined as

$$
\mathcal{A}=\left|\alpha_{m}\right|=\frac{1}{4 \eta k_{\beta}}\left|\frac{d \eta}{d s}\right|=\frac{1}{4 \eta^{3 / 2}}\left|\frac{d \eta}{d s}\right| \ll 1,
$$

which is equivalent to saying that the relative change in $\eta$ over a distance $k_{\beta}^{-1}$ is small. This makes no reference to the rate of change of the beam's beta function, only the rate of change of the plasma density.

The transverse dynamics of a single particle are described by the Hamiltonian,

$$
H=\frac{1}{2} x^{\prime 2}+\frac{1}{2} \eta x^{2}
$$

If the plasma density changes adiabatically, the evolution of the beam can be found using the theory of adiabatic invariants. The action-angle variables $J_{0}$ and $\theta_{0}$ in a uniform density plasma are generated by the function 


$$
F_{1}\left(x, \theta_{0}\right)=-\frac{x^{2}}{2 \beta_{m}} \tan \theta_{0}
$$

where the subscript 0 denotes that these variables are only action-angle variables for the Hamiltonian with constant $\eta$.

For a Hamiltonian with longitudinally varying plasma density, $J_{0}$ and $\theta_{0}$ are still valid canonical variables, but they have a dependence on $s$ given by

$$
\begin{aligned}
J_{0}^{\prime} & =-\beta_{m}^{\prime} \frac{\partial}{\partial \theta_{0}}\left(\frac{\partial F_{1}}{\partial \beta_{m}}\right) \\
\theta_{0}^{\prime} & =\frac{\partial H}{\partial J_{0}}+\beta_{m}^{\prime} \frac{\partial}{\partial J_{0}}\left(\frac{\partial F_{1}}{\partial \beta_{m}}\right) .
\end{aligned}
$$

Using the definition of $\alpha_{m}$ and taking the derivatives of the generating function gives expressions for $J_{0}^{\prime}$ and $\theta_{0}^{\prime}$, where the $s$ dependence of $\eta$ is a clear perturbation:

$$
J_{0}^{\prime}=2 \frac{\alpha_{m} J_{0}}{\beta_{m}} \cos 2 \theta_{0}, \quad \theta_{0}^{\prime}=\frac{1}{\beta_{m}}-\frac{\alpha_{m}}{\beta_{m}} \sin 2 \theta_{0} .
$$

These expressions can be expanded in powers of $\alpha_{m}$, the lowest order term for each coordinate is

$$
\theta_{0}^{(0)}=\int_{0}^{s} \frac{d \xi}{\beta_{m}(\xi)}, \quad J_{0}^{(0)}=J_{0}(0) .
$$

The coordinates can be used to find a transport matrix for single particle motion from which the adiabatic solution for the evolution of the $\beta$ function is found to be

$$
\beta=\beta_{m}\left(c_{0}+c_{1} \cos 2 \theta+c_{2} \sin 2 \theta\right),
$$

where $\theta=\theta_{0}^{(0)}$. The constants are functions of the initial conditions:

$$
\begin{aligned}
& c_{0}=\frac{1}{2}\left(\frac{\beta_{0}}{\beta_{m 0}}+\beta_{m 0} \gamma_{0}\right) \\
& c_{1}=\frac{1}{2}\left(\frac{\beta_{0}}{\beta_{m 0}}-\beta_{m 0} \gamma_{0}\right) \\
& c_{2}=-\alpha_{0},
\end{aligned}
$$

where $\beta_{m 0}=\beta_{m}(0)$. In the adiabatic limit the $\beta$ function oscillates with an amplitude proportional to the matched beam size about a value that is offset from, and proportional to, the matched beam size.

The first order solution is inconsistent in that $\beta^{\prime}(0) \neq-2 \alpha_{0}$. As a result, the solution does not include oscillations in the beta function that occur when $\alpha_{0}=0$ and $\alpha_{m 0} \neq 0$. It was pointed out by $\mathrm{Xu}$ et al. [11] that these oscillations would never appear in the lowest order adiabatic theory. As shown in the Appendix, a ramp with $\eta=1 /\left(1-2 \alpha_{m} s\right)^{2}$, which has constant $\mathcal{A}$, can be solved exactly. This solution can be used to derive a higher order correction to $c_{1}, c_{2}$, and $c_{3}$. The solution has the same form as Eq. (11), but with different constants. Expanding these constants to first order in $\alpha_{m}$ and inserting them into the adiabatic solution, captures the oscillations in $\beta$. This solution for the beta function corresponds to a transfer matrix where the second row is the derivative of the first. Working backwards it can be shown that the action variable must include some, but not all, of the second order terms. The higher order constants are given by

$$
\begin{aligned}
& c_{0}=\frac{1}{2}\left(\frac{\beta_{0}}{\beta_{m 0}}+\beta_{m 0} \gamma_{0}-2 \alpha_{0} \alpha_{m 0}\right) \\
& c_{1}=\frac{1}{2}\left(\frac{\beta_{0}}{\beta_{m 0}}-\beta_{m 0} \gamma_{0}+2 \alpha_{0} \alpha_{m 0}\right) \\
& c_{2}=-\alpha_{0}+\frac{\alpha_{m 0} \beta_{0}}{\beta_{m 0}} .
\end{aligned}
$$

The oscillations around the matched size disappear when $\beta_{0}=\beta_{m 0} / \sqrt{1+\alpha_{m 0}^{2}}$ and $\alpha_{0}=\alpha_{m 0} / \sqrt{1+\alpha_{m 0}^{2}}$. In the adiabatic limit this simplifies to $\beta_{0}=\beta_{m 0}$ and $\alpha_{0}=\alpha_{m 0}$. For a nonuniform plasma, we define this as the matched condition (this definition of matched was previously noted by Williams and Katsouleas [15]). If a beam is initially matched to an adiabatic plasma profile, the beam will remain matched to the plasma.

If it is assumed that $\beta_{0}=\beta_{m 0}$ and $\alpha_{0}=\alpha_{m 0}$, then very small oscillations of $\beta$ occur. Oscillations can also develop due to rapid changes in $\alpha_{m}$, even if the ramp remains adiabatic. Oscillations induced by discontinuous changes in $\alpha_{m}$ can be explained using the adiabatic solution with higher order constants. For example, if the gradient of the plasma density at the beginning of the exit ramp is discontinuous, a beam that is initially matched to the plasma in the accelerating region, where $\beta_{0}=1$ and $\alpha_{0}=0$, will not be matched to the exit ramp. Subsequently, it will undergo

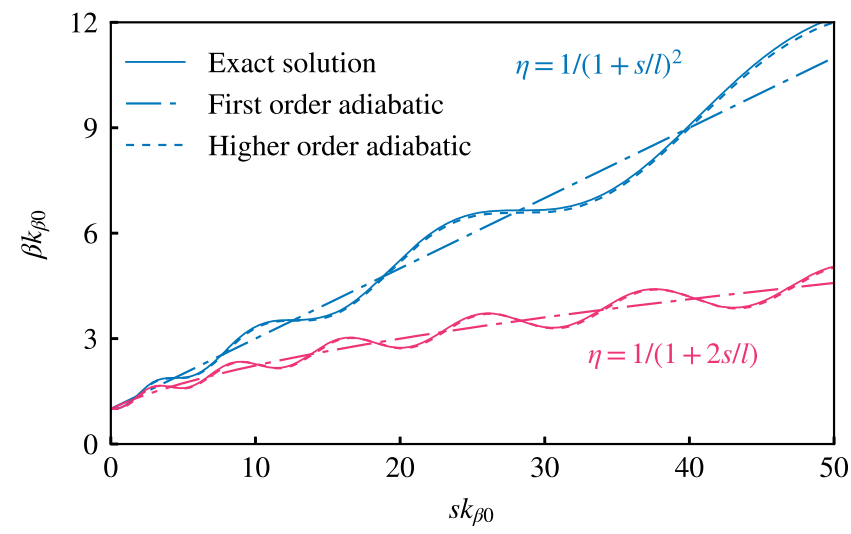

FIG. 3. Comparison of the lowest order adiabatic solution and the higher order adiabatic solution. Two different ramp profiles are portrayed, both with $l=5 / k_{\beta 0}$. The exact solution for the $\beta$ function evolution is shown as a thin solid line, the lowest order adiabatic solution as a dot-dashed line and the higher order solution as a dotted line. The higher order solution correctly predicts the oscillations of the beam around the matched $\beta$ function, which is equivalent to the first order adiabatic solution. 
oscillations. Examples of these oscillations for two exit ramps where the beta function can be found exactly are shown in Fig. 3. The ramp is identical to the primary ramp considered in [11], which has an exact solution for the beam evolution. As shown in the figure, the oscillations are not captured by the lowest order adiabatic solution, but they are captured by using the higher order correction to the constants.

Even if the reference energy is matched to the plasma, different energy slices of the beam will experience different focusing forces leading to projected emittance growth. Each energy component has a different matched beta function and will undergo adiabatic evolution according to Eq. (11). To lowest order in $\delta$, the projected emittance growth is a function of the beam's chromatic amplitude [18]:

$$
\frac{\Delta \epsilon}{\epsilon_{0}}=\frac{1}{2} W^{2} \sigma_{\delta}^{2},
$$

where $\sigma_{\delta}$ is the rms fractional momentum deviation, and the chromatic amplitude $W$ is defined as

$$
W^{2}=\left(\frac{\partial \alpha}{\partial \delta}-\frac{\alpha}{\beta} \frac{\partial \beta}{\partial \delta}\right)^{2}+\left(\frac{1}{\beta} \frac{\partial \beta}{\partial \delta}\right)^{2} .
$$

Here, all energy slices of the beam are assumed to start with the same geometric emittance $\epsilon_{0}$ and all the terms are evaluated at $\delta=0$. The expansion is only valid if $\beta(\delta)$ is approximately linear (even for small $\delta$ the quadratic term can sometimes be important).

It can be seen from Eq. (3) that $\beta(\delta)$ is given by making the replacement $\beta_{m} \rightarrow \beta_{m} \sqrt{1+\delta}$. Additionally, to account for any chromatic effects of the incoming beam, the initial conditions are functions of $\delta: \beta_{0}(\delta), \alpha_{0}(\delta)$ and $\gamma_{0}(\delta)$. Making the above substitution for $\beta_{m}, \beta_{m 0}$, and $\alpha_{m 0}$ gives

$$
\begin{aligned}
\beta(\delta)= & \beta_{m}\left[c_{3} \frac{\delta}{2}(1-\cos 2 \psi)+c_{2} \sqrt{1+\delta} \sin 2 \psi\right. \\
& \left.+c_{0}+c_{1} \cos 2 \psi\right]
\end{aligned}
$$

where $c_{3}=\beta_{m 0} \gamma_{0}-2 \alpha_{m 0} \alpha_{0}$, and

$$
\psi=\frac{\theta}{\sqrt{1+\delta}} .
$$

All the $c_{i}$ constants are functions of $\delta$ through $\beta_{0}, \alpha_{0}$, and $\gamma_{0} . \beta_{m}$ is the matched beta function for the average beam energy $\left\langle\gamma_{b}\right\rangle$. It is convenient to expand $\psi$ in $\delta$ : $\psi \approx(1-\delta / 2) \theta$. Evaluating the chromatic amplitude will give the lowest order emittance growth for a beam with arbitrary chromatic initial conditions.

Consider the case where all energy components start with the same initial conditions, and the reference energy is matched to the plasma. In this case the beam starts with its projected emittance equal to the emittance of each energy slice. $\beta(\delta)$ is given by

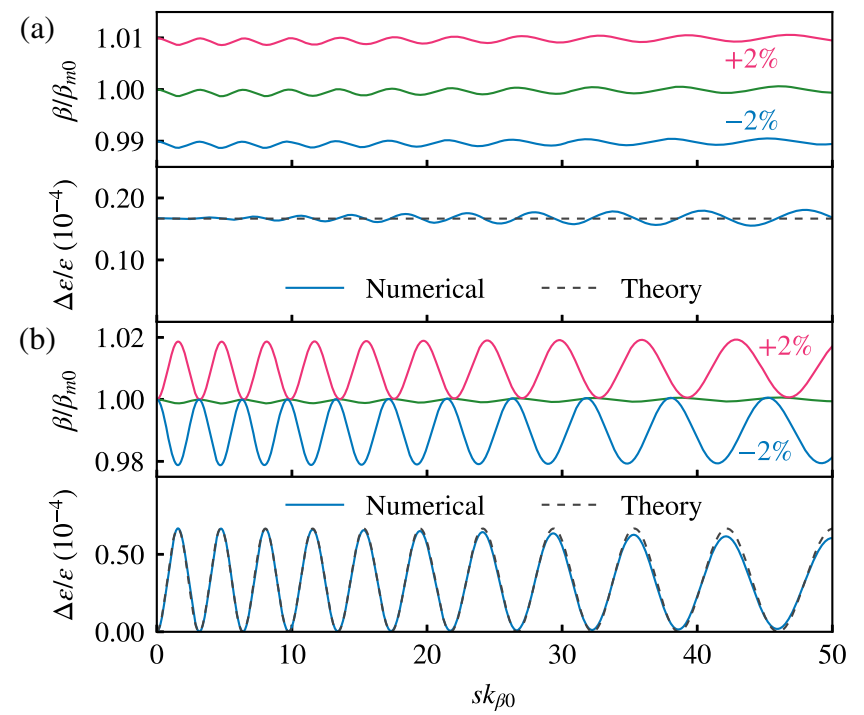

FIG. 4. Emittance evolution for a beam in an adiabatic plasma where (a) all energy components are matched, and (b) start with the same Courant-Snyder parameters. The beam has a uniform energy distribution from $-2 \%$ to $2 \%$ with the extremes of the distribution plotted. The plasma profile is $\eta=1 /\left[1+(a s)^{2}\right]$ with $a=0.05$.

$$
\beta(\delta)=\beta_{m}\left[\frac{\delta}{2}(1-\cos 2 \psi)+1\right] .
$$

Evaluating the chromatic amplitude shows that the emittance evolves according to

$$
\frac{\Delta \epsilon}{\epsilon_{0}}=\frac{1}{4}(1-\cos 2 \theta) \sigma_{\delta}^{2}
$$

to lowest order in $\delta$. This expression also holds true in a uniform plasma. The beam evolution and emittance evolution in an adiabatic plasma are shown in Fig. 4 for two different initial conditions. The model matches the numerical solution quite well in both cases. An oscillation of the projected emittance can occur so long as the emittance of each energy slice is smaller than the projected emittance of the entire beam. In the presence of nonuniform longitudinal fields, particle exchange between energy slices will lead to slice emittance growth and saturation of the projected emittance of the beam. The emittance will saturate to the maximum of the oscillation of the projected emittance. Figure 4(b) shows the emittance evolution for initial conditions where each energy slice is matched to the plasma. For most matched beams, the emittance growth in an adiabatic section would be negligible, on the order of $\sigma_{\delta}^{2}$.

If the reference energy is mismatched, $\beta(\delta)$ cannot be approximated as a linear function and the chromatic amplitude no longer predicts the emittance growth. The emittance growth in a uniform plasma from chromatic phase mixing has been found by [11] to be 


$$
\epsilon=\epsilon_{\text {sat }} \sqrt{1-\frac{\left(\gamma_{0} \beta_{m}+\beta_{0} / \beta_{m}\right)^{2}-4}{\left(\gamma_{0} \beta_{m}+\beta_{0} / \beta_{m}\right)^{2}}\left(\frac{\sin \Delta \psi}{\Delta \psi}\right)^{2}},
$$

where $\Delta \psi=\psi\left(\sqrt{3} \sigma_{\delta}\right)-\psi\left(-\sqrt{3} \sigma_{\delta}\right)$. The saturated emittance is given by $\epsilon_{\text {sat }}=\epsilon_{0}\left(\gamma_{0} \beta_{m}+\beta_{0} / \beta_{m}\right) / 2$, and represents the asymptotic upper limit of the emittance after many betatron oscillations. The phase mixing process works the same in an adiabatic plasma, so Eq. (20) can be used to describe the emittance growth of a mismatched beam in an adiabatic ramp.

It is useful to quantify the adiabatic ramp by the total phase advance in the ramp, $\theta_{f}$. The chromatic phase spread can then be written as $\Delta \psi \approx-\theta_{f} \delta$. Most ramps have $\theta_{f}$ on the order of $10 \mathrm{~s}$ of radians and $\Delta \Psi$ is small. Even for long adiabatic ramps, the emittance growth in the ramp is negligible. Adiabatic ramps are a robust option for matching a beam into a plasma. There is almost no emittance growth in the ramp if the beam is initially matched, and the exact shape of the ramp is inconsequential, so long as the plasma density changes slowly and $\mathcal{A}$ remains small.

\section{UNIFORM PLASMA RAMP}

Matching can be achieved in a very short distance by using a low density, uniform plasma in front of the accelerating plasma section. The electron beam enters the low density plasma mismatched and undergoes one quarter betatron oscillation, entering the accelerating plasma at the minimum of the oscillation where $\alpha=0$. An appropriate choice of plasma density ensures that $\beta=$ $\beta_{m}$ where the uniform plasma ramp meets the accelerating section. An example of such a ramp is shown in Fig. 5 where an $\eta=0.05$ density plasma produces a factor of 20 reduction in the $\beta$ function over a distance of $7 / k_{\beta 0}$.

The transverse beam evolution in the uniform density "ramp" is described exactly by the adiabatic theory and the results from the preceding section can be used to design the

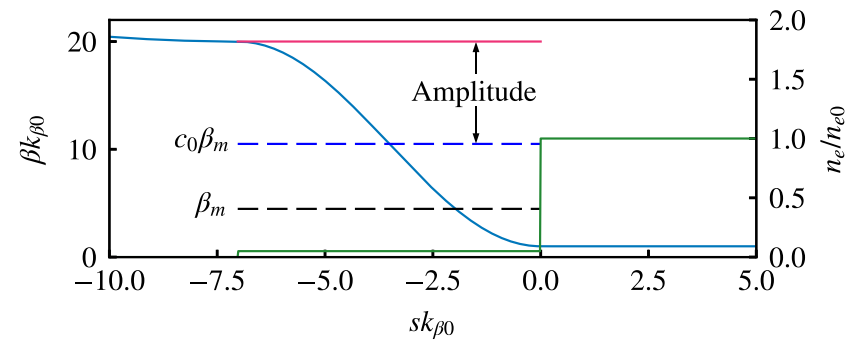

FIG. 5. A mismatched beam in a uniform density plasma ramp undergoes half an oscillation of $\beta$, rapidly focusing the beam. If the plasma ramp is truncated after a quarter betatron period it can be used to match the beam into a high density plasma in a short distance. The beam oscillates about a center value of $c_{0} \beta_{m}$, which is offset from the matched beam size $\beta_{m}$. The amplitude of the oscillation is given by $\sqrt{c_{1}^{2}+c_{2}^{2}}$. ramp. The plasma density required for focusing an incoming beam is given by

$$
\eta=\frac{1-\gamma_{0}}{\beta_{0}-1} .
$$

The necessary length of the ramp can be calculated by solving the phase equation

$$
\cos 2 \theta=\frac{2 \beta_{0}-1-\beta_{m}}{1-\beta_{m}},
$$

with $\beta_{m}$ determined from Eq. (21) and $\theta=L / \beta_{m}$, where $L$ is the length of the ramp.

If the beam is at a waist at the beginning of the ramp $\left(\alpha_{0}=0\right)$, then the length of the ramp must be $L=\pi /$ $(2 \sqrt{\eta})$. To the best of our knowledge, this is the shortest ramp yet considered in the literature. Very little chromatic phase spread develops in such a short ramp so the emittance growth is negligible. The ramp's length and low plasma density mitigate energy modulation of the witness beam before the accelerating section. Though the step function density profile of the theoretical model is nonphysical, the same qualitative behavior will be retained if the change in density occurs over a distance that is small compared to $k_{\beta}^{-1}$.

\section{NONADIABATIC EVOLUTION}

Ramps that are nonadiabatic, or have sections that are nonadiabatic, can focus beams over a much shorter distance than an adiabatic ramp with a matched beam. In general, the beam evolution in a nonadiabatic ramp cannot be solved for exactly; however, some ramp profiles have exact solutions that demonstrate the general character of the evolution. One such ramp is $\eta=1 /\left(1-2 \alpha_{m} s\right)^{2}$. The exact solution for the ramp is given in the Appendix. It is similar to the adiabatic solution except for a difference in constants and a correction to the phase. In the nonadiabatic regime it is impossible to choose a set of initial conditions such that the oscillating terms vanish for all $s$.

The fastest way to focus the beam in a nonadiabatic ramp is to harness the phase of the oscillation where the beam size is most rapidly decreasing. The nonadiabatic regime is unique in that the beam can undergo oscillations and still end up matched to the plasma. This is impossible in a smooth adiabatic ramp. In a well chosen nonadiabatic ramp, the beam undergoes almost exactly a quarter betatron oscillation and ends matched to the plasma. This is similar to the scheme presented in Sec. IV. The focusing process is shown in Fig. 6 where a nonadiabatic ramp is compared to an adiabatic ramp. The nonadiabatic ramp decreases the beta function by a factor of 30 in only $29 / k_{\beta 0}$ with the beam undergoing approximately a quarter of a betatron oscillation. An adiabatic ramp at the upper limit of the adiabatic regime $\left(\alpha_{m}=0.1\right.$ for the majority of the ramp) requires a distance of $152 / k_{\beta 0}$ to achieve the same degree of focusing. 


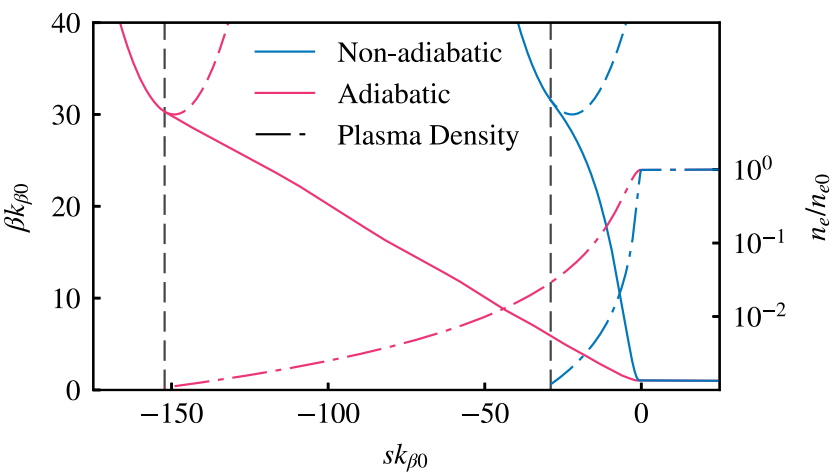

FIG. 6. Evolution of two identical beams with shifted vacuum waist locations through an adiabatic ramp (magenta) and a nonadiabatic ramp (blue). In the adiabatic ramp, the beam evolution follows the adiabatic solution, reducing $\beta$ by a factor of 30 in a distance of $152 k_{\beta 0}^{-1}$. In the nonadiabatic ramp, the beam undergoes a quarter of a betatron oscillation, reducing $\beta$ by a factor of 30 in $29 k_{\beta 0}^{-1}$. In both cases the beam ends matched to the uniform density acceleration section, with $\beta=1 / k_{\beta 0}$ and $\alpha=0$.

Generally, numerical methods must be implemented to design a smooth, matching ramp that provides rapid focusing. The ramp must transition from nonadiabatic to adiabatic exactly at the point where the beam oscillation passes through the matched solution. In practice, the matched Courant-Snyder parameters at the top of the nonadiabatic section can be numerically back propagated through a ramp of a given shape while scanning the characteristic ramp length until the ideal matching ramp length is identified.

Analytic solutions exist for ramps where $\beta_{m}(s)$ is linear or quadratic (see [10] for the quadratic case) which can be used to approximate the nonadiabatic part of an arbitrary ramp. Nonadiabatic ramps are sensitive to the exact ramp shape due to the interplay between oscillation and matching. These difficulties make nonadiabatic ramps potentially difficult to design and implement in situations where the plasma density cannot be precisely controlled.

\section{PERTURBATIVE PLASMA RAMP}

If the plasma has a sufficiently low density, the motion arising from the focusing force of the ion column can be treated as a perturbation to the vacuum propagation of the beam. The unperturbed Hamiltonian is the free space Hamiltonian and it is well known that the canonical transformation obtained using Hamilton's principle function results in the coordinates given by

$$
A=x_{0}^{\prime} \quad B=x-A s .
$$

According to classical, time dependent perturbation theory, the time evolution of these coordinates is given by

$$
\dot{A}=-\eta(s)(A s+B) \quad \dot{B}=\eta(s) s(A s+B) .
$$

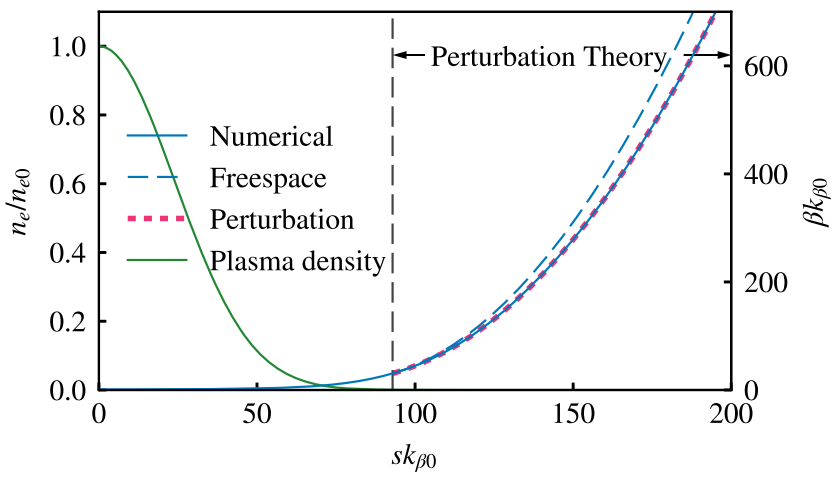

FIG. 7. Evolution of an initially matched beam in an exit ramp with a long perturbative tail. The perturbative theory effectively models the small deviation in $\beta$ from free space evolution caused by the long, low density tail of a Gaussian plasma density ramp (green).

The zeroth order solution is the constant solution of the unperturbed Hamiltonian: $A_{0}=x_{0}^{\prime}$ and $B_{0}=x_{0}$. The first order solution is found by inserting the zeroth order solution into the right-hand side of the differential equation and integrating. The first order solution can be written in the form of a transport matrix for $x$ and $x^{\prime}$ from $s=0$ to $s$ :

$$
M=\left(\begin{array}{cc}
1-I_{0} s+I_{1} & s-I_{1} s+I_{2} \\
-I_{0} & 1-I_{1}
\end{array}\right),
$$

where $I_{n}$ refers to integrals given by

$$
I_{n}=\int_{0}^{s} \zeta^{n} \eta(\zeta) d \zeta
$$

The approximation is valid if $\frac{1}{2} \eta x^{2} \ll \frac{1}{2} x^{\prime 2}$, requiring that $\beta k_{\beta}^{2} / \gamma \ll 1$. The $\beta$ function evolution can be derived in the normal way from the components of the transport matrix. Figure 7 shows that the approximation well represents the beam evolution in the low density tail of an exit ramp with a Gaussian density profile.

As $s$ becomes very large, the integrals converge for any practical ramp. Beyond this point, the plasma has no effect on the beam and the beam evolves as it would in vacuum. The beam will appear to have come from a vacuum waist of $\beta^{*}=1 / \gamma$ :

$\beta^{*}=\frac{1}{\gamma_{0}+2\left(\alpha_{0} I_{0}-\gamma_{0} I_{1}\right)+\beta_{0} I_{0}^{2}-2 \alpha_{0} I_{0} I_{1}+\gamma_{0} I_{1}^{2}}$,

where the $I_{n}$ integrals are assumed to be convergent and evaluated to infinity. After the beam has left the perturbative region of an exit ramp, $\beta^{*}$ will be the effective waist seen by the downstream magnetic focusing optics. For an entrance ramp, this formula can be used to calculate the focusing of the beam that occurs at the foot of the ramp. It can be useful to drop terms of order $I^{2}$ to get a compact version of the formula. 
It is straightforward to account for chromatics in the perturbative ramp, a factor of $1 /(1+\delta)$ appears in front of each $I_{n}$ integral. The transport matrix for off energy particles can easily be found and $\beta(\delta)$ solved for. The chromatic amplitude gives emittance growth induced in the perturbative part of the ramp, which will be negligible in most cases. The net effect of the perturbative ramp on the motion does not depend on the exact shape of the ramp, only the values of the integrals $I_{n}$ evaluated over the entire ramp.

\section{EXAMPLE RAMP DESIGN}

Here, we demonstrate how to use adiabatic and perturbative plasma regions to match a beam originating from a conventional magnetic delivery system into a plasma accelerator. Using these two regions, the beam dynamics can be solved for analytically. In addition, the ramp is robust against errors in the plasma density profile. We assume in this example that the magnetic optics can only focus the beam down to $\beta^{*}=10 k_{\beta 0}^{-1}$ in vacuum. The plasma ramp must demagnify the $\beta$ function by a factor of 10 for the beam to be matched to the uniform density, accelerating plasma section. The challenge is to determine an appropriate ramp shape as well as the location of the vacuum focus $s^{*}$ for the incoming beam.

We will use an adiabatic ramp that is truncated on the upstream side by a short perturbative section. The adiabatic section accomplishes most of the beam focusing and the small perturbative section rapidly brings the plasma density up from zero at the start of the ramp. In the adiabatic section, a plasma density given by $\eta(s)=1 /\left[1+(a s)^{2}\right]$ is used because it has a smooth profile at the top and constant $\alpha_{m}$ in the bulk of the ramp, minimizing the length of the ramp. The perturbative region has a Gaussian plasma density profile given by $\eta(s)=\eta_{t} \exp \left[-\left(s-s_{t}\right)^{2} / 2 \sigma^{2}\right]$, where $s_{t}$ is the location of the transition between the perturbative and adiabatic section and $\eta_{t}$ is the plasma density at that point. The length scale of the Gaussian is set by $\sigma$. A Gaussian profile is chosen to make evaluating the integrals in the perturbative section of the ramp straightforward. In the adiabatic section, the beam undergoes adiabatic evolution and remains matched to the plasma $\beta=\beta_{m}$. To focus the beam by a factor of 10 the plasma density must increase from $\eta=1 / \beta_{0}^{2}$ to $\eta=1$. To keep the ramp as short as possible, the maximum $\mathcal{A}$ is chosen to be $0.1(a=0.2)$, which is the approximate upper limit for accurate predictions using the adiabatic approximation. Some focusing will occur in the perturbation section of the ramp so the plasma density increases adiabatically from $\eta=\eta_{t}=0.012$ to $\eta=1$. The lower bound on the plasma density was chosen somewhat arbitrarily to create a perturbative section of reasonable length. In this perturbative section $\beta$ decreases by $8 \%$ with respect to $\beta^{*}$.

The length of the adiabatic section is determined by solving $\eta_{t}=1 /\left[1+\left(a s_{t}\right)^{2}\right]$ for $s_{t}$, giving a length of $s_{t}=-45.4 k_{\beta 0}^{-1}$, where $s=0$ is chosen to be at the top of the ramp. The next step is to determine the length of the perturbative section and the location of the incoming beam's vacuum waist. At the beginning of the adiabatic section the Courant-Snyder parameters must be $\alpha=\alpha_{m}=$ 0.1 and $\beta=\beta_{m}=1 / \sqrt{\eta}=9.13 k_{\beta 0}^{-1}$. The length of the perturbation section can be determined by solving Eq. (27) for $\sigma$. The integrals are taken from 0 to $-\infty$, effectively going backwards from $s_{t}$. Using this, the perturbative section needs to have $\sigma=2.645 k_{\beta 0}^{-1}$ to match a $\beta^{*}=10 k_{\beta 0}^{-1}$ beam into the adiabatic section of the plasma. Next, the transport matrix for the perturbation section can be calculated to find the beam waist. Because the integrals were calculated in the negative direction, the transport matrix propagates in the negative direction. The beam waist can be determined by back propagating the beta function from the end of the perturbative ramp into free space and solving for the vacuum waist from the free space values. Using this method, it is found that the vacuum waist should be placed at $s=-48.04 k_{\beta 0}^{-1}$.

The emittance evolution in the ramp can be found using the chromatic amplitude. As an example, we start with an incoming beam with a uniform energy distribution between $1 \%$ and $-1 \%$. All energy components focus to the same $\beta$ at the vacuum waist, but $\alpha$ at the waist depends linearly on energy $\alpha\left(s^{*}\right)=r \delta$. Here, $r$ is an arbitrary parameter representing the chromatics in the incoming beam. The $\delta$ dependent transport matrix for the perturbative section can be used to find the beam's initial state at the beginning of the adiabatic section. The $\delta$ dependent adiabatic solution can be used to find the chromatic amplitude, and thus the emittance growth, at all points in the adiabatic section. Figure 8 shows the beam evolution in the plasma for an incoming beam with $r=5.5$. The centroid energy slice does not display any significant oscillations about the matched size, demonstrating that the beam is correctly matched to the adiabatic section of the ramp. The off energy components undergo significant oscillations in the ramp, but the ramp itself has negligible impact on the beam emittance. The initial projected emittance is larger than the slice emittance due to the chromatics in the beam before it reaches the plasma ramp.

The theoretical calculation carried out in this example shows good agreement with the numerical solution for both the transverse dynamics and the emittance evolution. The theory presented here provides a simple framework with which to design and evaluate plasma ramps in order to preserve beam quality.

A ramp with an identical functional form can be used to control the divergence of the beam at the exit of the plasma based accelerator. At the exit, however, the beam energy is greater than at the entrance. This results in a decrease of $k_{\beta 0}$ for the exit ramp, causing an increase in the length scale of the ramp. If the density of the accelerating region remains 


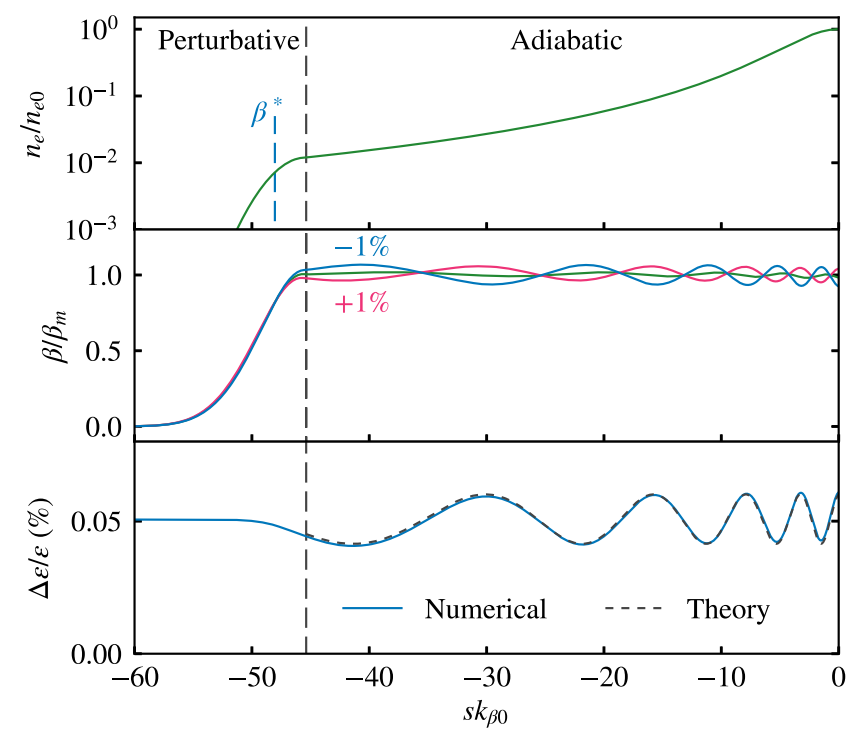

FIG. 8. An example of a perturbative and adiabatic ramp designed to focus a beam into an accelerating plasma. The incoming beam is assumed to focus all energy components to the same size at the vacuum waist. The length of the perturbation region and the incoming beam's vacuum waist location were determined using the ramp theory presented in this paper. The emittance evolution of the beam is well described by the analytic solution found using the chromatic amplitude function.

fixed, the ramp length increases by a factor of $\sqrt{\gamma_{b f} / \gamma_{b i}}$, where the subscripts $i$ and $f$ denote preacceleration and postacceleration, respectively. After traversing the exit ramp, the beam would behave as if it originated from a vacuum waist with $\beta^{*}=\beta_{0}^{*} \sqrt{\gamma_{b i} / \gamma_{b f}}$, where $\beta_{0}^{*}$ is the vacuum waist produced by the upstream magnetic focusing optics.

\section{CONCLUSIONS}

A tailored longitudinal plasma density profile can be used to match a beam into or out of a plasma based accelerator in order to minimize chromatic emittance growth in the beam. The length of the ramp, location of the vacuum waist, and emittance growth in the ramp can be calculated analytically for a large range of ramp shapes by separating arbitrary plasma profiles - or portions thereofinto three qualitatively distinct types. The first is an adiabatic plasma ramp where the plasma density changes slowly. In this type of ramp, a beam that starts out matched to the plasma remains matched to the plasma and undergoes minimal emittance growth. Adiabatic ramps are long but insensitive to the exact form of the density profile, so long as the gradient of the plasma density remains small. The second is a nonadiabatic plasma ramp where the beam is not matched to the plasma and undergoes a fraction of an oscillation. This type of plasma ramp can focus the beam quickly, but the beam dynamics are sensitive to the exact shape of the plasma density profile. The third is a perturbative plasma ramp where the plasma density is low and the beam evolution is only slightly perturbed from vacuumlike propagation. This type of ramp is somewhat insensitive to the form of the plasma density profile but can only cause minimal focusing of the beam. The beam dynamics can be calculated exactly for the adiabatic and perturbative ramp. The beam dynamics in a nonadiabatic section can be calculated numerically or approximated by a ramp where the dynamics are analytically solvable.

A wide range of ramp shapes can match the same beam into a plasma based accelerator, but different density profiles can affect the focusing process. The fastest focusing can be achieved with a subset of the adiabatic plasma ramps, wherein a highly mismatched beam undergoes a quarter of a betatron oscillation in a low density uniform plasma. In contrast, adiabatic ramps are long, but are insensitive to errors in the plasma density profile. Multiple ramp types can be combined to produce a ramp that suits a particular set of experimental needs. The theoretical work presented here can be used to compare different ramp shapes by studying the sensitivity of the emittance growth to the initial beam conditions and variations in the plasma density profile. This can be extended to include the effects of nonuniformity in the accelerating section of the plasma.

To form a complete description of the beam evolution in the wake, the longitudinal dynamics should also be included in the analysis. In a short ramp, the beam does not interact with the longitudinal electric field in the ramp for very long, limiting the effect it has on the beam's total energy and energy spread. In a longer ramp, however, the loading of the wake and the changing phase of the witness beam will play an important role in the beam's energy distribution. This, in turn, will affect the transverse chromatic dynamics of the beam throughout the plasma. Future studies should examine the longitudinal dynamics of the beam to determine how much of an effect these dynamics have as the length of the ramp increases.

\section{ACKNOWLEDGMENTS}

This material is based upon work supported by the U.S. Department of Energy, Office of Science, Office of High Energy Physics under Award No. DE-SC0017906.

\section{APPENDIX: SOLUTION FOR THE BETA FUNCTION EVOLUTION}

Taking the derivative of Eq. (3) gives a linear third order differential equation describing the evolution of $\beta$ :

$$
\beta^{\prime \prime \prime}+4 \eta \beta^{\prime}+2 \eta^{\prime} \beta=0 .
$$

Using the definition of $\beta_{m}$ the equation can be written as

$$
\beta_{m}^{3} \beta^{\prime \prime \prime}+4 \beta_{m} \beta^{\prime}+8 \alpha_{m} \beta=0
$$


If $\beta_{m}=\beta_{m 0}-2 \alpha_{m 0} s$, corresponding to a plasma density of $\eta=1 /\left(\beta_{m 0}-2 \alpha_{m} s\right)^{2}$, then the equation is a Cauchy-Euler equation. Changing variables to $\beta_{m}$ makes this obvious:

$$
\beta_{m}^{3} \alpha_{m 0}^{3} \frac{d^{3} \beta}{d \beta_{m}^{3}}+\beta_{m} \alpha_{m 0} \frac{d \beta}{d \beta_{m}}-\alpha_{m 0} \beta=0 .
$$

The solution has the form

$$
\beta=C_{n} \beta_{m}^{n},
$$

where $C_{n}$ is an arbitrary constant and $n$ is a solution of the equation

$$
(n-1)\left[\alpha_{m 0}^{2} n(n-2)+1\right]=0 .
$$

The beta function evolves according to

$$
\beta=\beta_{m}\left(c_{0}+c_{+} e^{i 2 \Phi}+c_{-} e^{-i 2 \Phi}\right),
$$

where

$$
\Phi=\frac{1}{2 \alpha_{m}} \sqrt{1-\alpha_{m}^{2}} \ln \beta_{m} .
$$

Insisting that the solution is real constrains the coefficients such that $c_{0}$ is real and $c_{+}^{*}=c_{-}$. We can define a new pair of coefficients as $c_{ \pm}=\left(c_{1} \pm i c_{2}\right) / 2$. The evolution is now written in the familiar form,

$$
\beta=\beta_{m}\left(c_{0}+c_{1} \cos 2 \Phi+c_{2} \sin 2 \Phi\right) .
$$

The third order differential equation introduces an additional constant that can be removed by requiring that the solution satisfy Eq. (3). After some algebra it is found that $c_{0}$ is a function of $c_{1}$ and $c_{2}$ :

$$
c_{0}=\sqrt{c_{1}^{2}+c_{2}^{2}+\frac{1}{1-\alpha_{m}^{2}}} .
$$

Letting $\beta_{m 0}=1$, the constants $c_{1}$ and $c_{2}$ are given in terms of the initial Courant-Snyder parameters at $s=0$ as

$$
\begin{gathered}
c_{1}=\frac{1}{2}\left(\beta_{0}-\frac{\gamma_{0}-2 \alpha_{0} \alpha_{m 0}+\alpha_{m 0}^{2} \beta_{0}}{1-\alpha_{m 0}^{2}}\right), \\
c_{2}=\frac{\alpha_{0}-\alpha_{m 0} \beta_{0}}{\sqrt{1-\alpha_{m 0}^{2}}} .
\end{gathered}
$$

The constants reduce to the adiabatic solution when $\alpha_{m 0} \rightarrow 0$.

[1] I. Blumenfeld, C. E. Clayton, F.-J. Decker, M. J. Hogan, C. Huang, R. Ischebeck, R. Iverson, C. Joshi, T. Katsouleas, N. Kirby, W. Lu, K. A. Marsh, W. B. Mori, P. Muggli, E. Oz, R. H. Siemann, D. Walz, and M. Zhou, Energy doubling of $42 \mathrm{GeV}$ electrons in a metre-scale plasma wakefield accelerator, Nature (London) 445, 741 (2007).
[2] M. Litos et al., High-efficiency acceleration of an electron beam in a plasma wakefield accelerator, Nature (London) 515, 92 (2014).

[3] M. Litos, E. Adli, J. M. Allen, W. An, C. I. Clarke, S. Corde, C. E. Clayton, J. Frederico, S. J. Gessner, S.Z. Green, M. J. Hogan, C. Joshi, W. Lu, K. A. Marsh, W. B. Mori, M. Schmeltz, N. Vafaei-Najafabadi, and V. Yakimenko, $9 \mathrm{GeV}$ energy gain in a beam-driven plasma wakefield accelerator, Plasma Phys. Controlled Fusion 58, 034017 (2016).

[4] W. P. Leemans, B. Nagler, A. J. Gonsalves, C. Tóth, K. Nakamura, C. G. Geddes, E. Esarey, C. B. Schroeder, and S. M. Hooker, GeV electron beams from a centimetre-scale accelerator, Nat. Phys. 2, 696 (2006).

[5] W. P. Leemans, A. J. Gonsalves, H. S. Mao, K. Nakamura, C. Benedetti, C. B. Schroeder, C. Tóth, J. Daniels, D. E. Mittelberger, S. S. Bulanov, J. L. Vay, C. G. R. Geddes, and E. Esarey, Multi-GeV Electron Beams from Capillary-Discharge-Guided Subpetawatt Laser Pulses in the Self-Trapping Regime, Phys. Rev. Lett. 113, 245002 (2014).

[6] P. Antici, A. Bacci, C. Benedetti, E. Chiadroni, M. Ferrario, A. R. Rossi, L. Lancia, M. Migliorati, A. Mostacci, L. Palumbo, and L. Serafini, Laser-driven electron beamlines generated by coupling laser-plasma sources with conventional transport systems, J. Appl. Phys. 112, 044902 (2012).

[7] M. Migliorati, A. Bacci, C. Benedetti, E. Chiadroni, M. Ferrario, A. Mostacci, L. Palumbo, A. R. Rossi, L. Serafini, and P. Antici, Intrinsic normalized emittance growth in laser-driven electron accelerators, Phys. Rev. ST Accel. Beams 16, 011302 (2013).

[8] T. Mehrling, J. Grebenyuk, F. S. Tsung, K. Floettmann, and J. Osterhoff, Transverse emittance growth in staged laserwakefield acceleration, Phys. Rev. ST Accel. Beams 15, 111303 (2012).

[9] K. A. Marsh, C. E. Clayton, D. K. Johnson, C. Huang, C. Joshi, W. Lu, W. B. Mori, M. Zhou, C. D. Barnes, F. J. Decker, M. J. Hogan, R. Iverson, P. Krejcik, C. L. O’Connell, R. Siemann, D. Walz, S. Deng, T. C. Katsouleas, P. Muggli, and E. Oz, Beam matching to a plasma wake field accelerator using a ramped density profile at the plasma boundary, in Proceedings of the IEEE Particle Accelerator Conference (IEEE, New York, 2005), Vol. 2005, pp. 2702-2704.

[10] K. Floettmann, Adiabatic matching section for plasma accelerated beams, Phys. Rev. ST Accel. Beams 17, 054402 (2014).

[11] X. L. Xu, J. F. Hua, Y. P. Wu, C. J. Zhang, F. Li, Y. Wan, C. H. Pai, W. Lu, W. An, P. Yu, M. J. Hogan, C. Joshi, and W. B. Mori, Physics of Phase Space Matching for Staging Plasma and Traditional Accelerator Components Using Longitudinally Tailored Plasma Profiles, Phys. Rev. Lett. 116, 124801 (2016).

[12] P. Chen, K. Oide, A. M. Sessler, and S. S. Yu, PlasmaBased Adiabatic Focuser, Phys. Rev. Lett. 64, 1231 (1990).

[13] D. Dimitrov, R. Giacone, D. Bruhwiler, R. Busby, J. Cary, C. Geddes, E. Esarey, and W. Leemans, Coupling of laser energy into plasma channels, Phys. Plasmas 14, 043105 (2007). 
[14] S. Lee, Accelerator Physics, 3rd ed. (World Scientific Publishing Co. Pte. Ltd., Hackenensack, 2012).

[15] R. L. Williams and T. Katsouleas, Numerical studies on the ramped density plasma lens, in AIP Conference Proceedings (AIP, New York, 1992), Vol. 279, pp. 565-578.

[16] N. Barov and J.B. Rosenzweig, Propagation of short electron pulses in underdense plasmas, Phys. Rev. E 49, 4407 (1994).
[17] I. Dornmair, K. Floettmann, and A. R. Maier, Emittance conservation by tailored focusing profiles in a plasma accelerator, Phys. Rev. ST Accel. Beams 18, 041302 (2015).

[18] C. A. Lindstrøm and E. Adli, Design of general apochromatic drift-quadrupole beam lines, Phys. Rev. Accel. Beams 19, 071002 (2016). 